\title{
Antioxidative Property of Acyl Ascorbate in Cookies Containing Iron
}

\author{
Yoshiyuki WATANABE ${ }^{1, \dagger}$, Pramote KHUWIJITJARU ${ }^{2,3}$, Yoshie HAMAI $^{1}$, \\ Jintana WIBOONSIRIKUL ${ }^{4}$, Shuji ADACHI ${ }^{2}$ \\ ${ }^{1}$ Department of Biotechnology and Chemistry, Faculty of Engineering, Kindai University, \\ 1 Umenobe, Takaya, Higashi-Hiroshima 739-2116, Japan \\ ${ }^{2}$ Division of Food Science and Biotechnology, Graduate School of Agriculture, Kyoto University, \\ Sakyo-ku, Kyoto 606-8502, Japan \\ ${ }^{3}$ Department of Food Technology, Faculty of Engineering and Industrial Technology, Silpakorn University, \\ Nakhon Pathom 73000, Thailand \\ ${ }^{4}$ Department of Food Science and Technology, Faculty of Agricultural Technology, Phetchaburi Rajabhat University, \\ Phetchaburi 76000, Thailand
}

\begin{abstract}
Acyl ascorbates are ascorbic acid derivatives, which are possibly used as antioxidants in oil systems. The effect of stearoyl ascorbate at different amounts in model cookies containing iron, in terms of lipid autoxidation, was studied. A kinetic model describing the formation and degradation of lipid hydroperoxides (expressed as peroxide value) was developed to compare the antioxidant abilities. Results showed that addition of $25 \mathrm{mg}$ of stearoyl ascorbate to $250 \mu \mathrm{L}$ of soybean oil resulted in the longest induction time among the tested systems. Additionally, stearoyl ascorbate showed much higher antioxidant ability compared to palmitoyl and behenoyl ascorbates.
\end{abstract}

Keywords: acyl ascorbate; cookies; iron; lipid oxidation; peroxide value

\section{Introduction}

Rancidity is a major factor contributing to the deterioration of food containing fat and oil, because autoxidation of unsaturated fatty acids is a thermodynamically favorable reaction that proceeds at a considerable rate even at low temperatures. However, retardation of lipid oxidation can be achieved by several methods. Removal of oxygen, protection from light, and addition of antioxidants are among the several methods routinely applied in the food industries [1] to prolong the shelf-life of food products and prevent rancidity.

Acyl ascorbates, which are esters of fatty acids with different chain lengths and ascorbic acid, have been found to be effective antioxidants for bulk, disperse, and microcapsule oil systems [2]. The acyl chain makes it possible to use ascorbic acid in oil systems. Currently, palmitoyl and stearoyl ascorbates are permitted for use as food additives. Aleman et al. [3] reported that palmitoyl ascorbate at $0.04 \%(\mathrm{w} / \mathrm{w})$ effectively suppressed tocopherol loss and oxidation of chocolate cream used in sandwich-type cookies, while Zou and Akoh [4] found

(Received 12 Mar. 2016: accepted 2 Jun. 2016)

$\dagger$ Fax: 082-434-7890, E-mail: wysyk@hiro.kindai.ac.jp that palmitoyl ascorbate at $0.005 \%(\mathrm{w} / \mathrm{v})$ is the most effective antioxidant in infant formula emulsion as compared to $\alpha$-tocopherol, $\beta$-carotene, ascorbic acid, and citric acid.

The antioxidative properties of antioxidants in simple systems, such as bulk oil [5], emulsions containing a single oil and single aqueous component [6], encapsulated oil with a single wall material [7], have been evaluated to gain basic information regarding antioxidative properties of acyl ascorbates. However, to determine the applicability of antioxidants in food products, a more complex, controlled food model should be tested. Therefore in this study, model cookies containing iron, a lipid autoxidation catalyst, were used to test the efficiency of long-chain acyl ascorbates for the retardation of lipid autoxidation, by kinetic analysis of the peroxide value.

\section{Materials and Methods}

\subsection{Materials}

Stearoyl ascorbate, palmitoyl ascorbate, and soybean oil were purchased from Wako Pure Chemical Industries (Tokyo, Japan). Behenic acid was obtained from Kishida Chemicals (Osaka, Japan). Ascorbic acid was obtained from Nacalai Tesque (Kyoto, Japan). Palmitoyl sucrose 
ester (P-1670) was purchased from Mitsubishi Kagaku, (Tokyo, Japan). Immobilized lipase from Candida antarctica (Chirazyme ${ }^{\circledR} \mathrm{L}-2$ C2) was provided by Roche (Mannheim, Germany). Commercial wheat flour (Nisshin Flour Milling), unsalted, no vitamin E added butter (Megmilk Snow Brand), white sugar (Pearl Ace), and table salt (Ajinomoto) were used for preparing the model cookies. Other reagents used were of analytical grade.

\subsection{Preparation of behenoyl ascorbate}

Because behenoyl ascorbate is not commercially available, it was prepared by enzymatic reaction under the optimal conditions previously reported [8]. $15 \mathrm{mmol}$ of ascorbic acid, $45 \mathrm{mmol}$ of behenic acid, $1 \mathrm{~g}$ of Chirazyme $^{\circledR} \mathrm{L}-2 \mathrm{C} 2$, and $300 \mathrm{~mL}$ of acetone were combined in an amber glass bottle and briefly mixed in a shaking water bath $(150 \mathrm{rpm})$ at $50^{\circ} \mathrm{C}$ for $24 \mathrm{~h}$ to allow the reaction to proceed.

Behenoyl ascorbate was purified by filtering the reaction mixture through Advantec No. 1 filter paper. The filtrate was concentrated in a vacuum evaporator at $50^{\circ} \mathrm{C}$. The concentrated solution was combined with hexane, filtered, and then washed with hexane several times to remove all unreacted fatty acid. The obtained solid residue was transferred to a $500 \mathrm{~mL}$ separating funnel and combined with $100 \mathrm{~mL}$ of 2-butanol and $50 \mathrm{~mL}$ of distilled water to partition the product into the 2-butanol phase and the unreacted ascorbic acid into water. The lower water phase was then discarded. The 2-butanol phase was repeatedly washed with water several times for the complete removal of ascorbic acid. Then, butanol was removed by evaporating under vacuum at $50^{\circ} \mathrm{C}$. The final product was further dried in a desiccator that was kept in the dark, together with phosphorus pentoxide, under vacuum for one day. The purity of the product was checked by HPLC, and the dried product was kept at $4^{\circ} \mathrm{C}$.

\subsection{Preparation of cookies containing iron}

Cookies containing iron were prepared as a model food for studying the antioxidative property of acyl ascorbates. Wheat flour (25 g), white sugar $(12.5 \mathrm{~g})$, melted butter $(12.5 \mathrm{~g})$, soybean oil $(250 \mu \mathrm{L})$, and table salt $(0.1875 \mathrm{~g})$ were mixed well in a bowl. Distilled water (2.5 $\mathrm{mL})$, iron (II) chloride tetrahydrate $\left(\mathrm{FeCl}_{2} \cdot 4 \mathrm{H}_{2} \mathrm{O} ; 25\right.$ $\mathrm{mg}$ ), and the specific amount of acyl ascorbate or ascorbic acid were mixed in a test tube and added to the bowl. The mixture was then formed into dough. The dough was flatted with a roller, wrapped in plastic wrap, and chilled in a refrigerator for $10 \mathrm{~min}$. The refrigerated dough was cut into flat circles with a diameter of $1.5 \mathrm{~cm}$ and baked in an electric oven at $180^{\circ} \mathrm{C}$ for $12 \mathrm{~min}$.

\subsection{Measurement of the peroxide values of cookies}

The prepared cookies with different added amounts of the test components, i.e. palmitoyl ascorbate (C16AsA), stearoyl ascorbate (C18AsA), behenoyl ascorbate (C22AsA), ascorbic acid with palmitoyl sucrose ester (AsA+SEC16 at a ratio of 1:1 by weight), and palmitoyl sucrose ester (SEC16), were tested for their ability to retard lipid autoxidation. Each group of cookies was kept in a plastic box at a relative humidity of $44 \%$ adjusted by a saturated solution of potassium carbonate and controlled at $65^{\circ} \mathrm{C}$ by placing them in a hot-air incubator. A piece of cookie, which contained $c a .194 \mathrm{mg}$ of lipid, was taken at appropriate time intervals, ground in a mortar and pestle, and extracted for oil with chloroform/methanol (2:1 $\mathrm{v} / \mathrm{v}$ ). The peroxide value (PV) of the extracted oil was spectrophotometrically measured according to the method proposed by Løvaas [9] and reported as meq/ $\mathrm{kg}$-oil. Each measurement was done in duplicate, and the mean value was calculated.

\section{Results and Discussion}

\subsection{Effect of amount of stearoyl ascorbate on the oxidation of soybean oil in cookies containing iron}

Figure 1 shows changes in PV with time for the cookies with 5, 25, and $100 \mathrm{mg}$ of C18AsA. SEC16 (25 mg) was used as a control because it contained the acyl chain but possesses no antioxidant activity. PV represents the amount of lipid hydroperoxides, which are the primary products of lipid oxidation and are widely used as an indicator of the extent of lipid oxidation in food products at early stages. It is known that PV usually increases with time, after a certain period of time called induction period of lipid oxidation, but decreases at some point because hydroperoxides are unstable and further degrade to other products. [10]

The quantitative kinetic model of autoxidation is very useful to analyze the effectiveness of each antioxidant on the retardation of lipid oxidation. Iibuchi [11] proposed a comprehensive model for the autoxidation of edible oil to describe the change in PV, but for the purpose of this study, a simple kinetic model for autoxidation was 


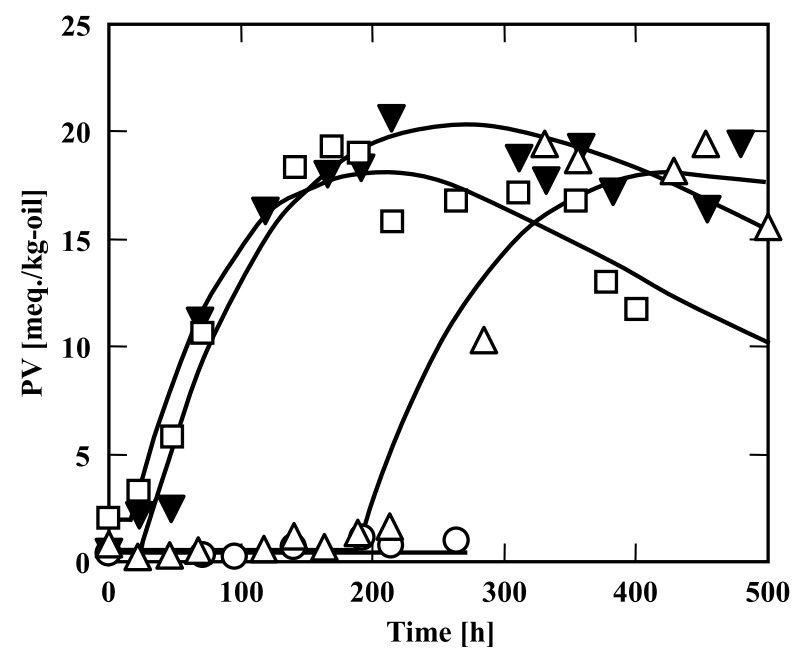

Fig. 1 Changes in peroxide values (PV) of soybean oil (250 $\mu$ L) with time for the cookies with $(\square) 5$, $(\bigcirc) 25$, and $(\triangle) 100$ $\mathrm{mg}$ of stearoyl ascorbate (C18AsA). The change in the PV of linoleic acid for the cookie added with palmitoyl sucrose ester (SEC16) ( $\boldsymbol{\nabla}$ ) was observed as a control. The solid curves were calculated using the estimated kinetic parameters.

adopted:

$$
\mathrm{S} \stackrel{k_{1}}{\longrightarrow} \mathrm{PV} \stackrel{k_{2}}{\longrightarrow} \text { other products }
$$

where $\mathrm{S}$ is the concentration of the lipid substrate, which produces peroxide through oxidation (meq/ $\mathrm{kg}-\mathrm{oil})$, PV is the peroxide value (meq/ $\mathrm{kg}-\mathrm{oil}$ ), and $k_{1}$ and $k_{2}$ are the assumed first-order kinetic reaction rate constants for the formation and degradation of hydroperoxides, respectively $\left(\mathrm{h}^{-1}\right)$. Because lipid oxidation normally proceeds after an induction time $\left(t_{0}\right)$, we could estimate the value of $\mathrm{PV}$ at any time $(t)$ from

$$
\begin{gathered}
\mathrm{PV}=\mathrm{PV}_{0} \text { at } t<t_{0} \\
\mathrm{PV}=\frac{\mathrm{S}_{0}-\mathrm{PV}_{0}}{1-k_{2} / k_{1}}\left[e^{-k_{2}\left(t-t_{0}\right)}-e^{-k_{1}\left(t-t_{0}\right)}\right]+\mathrm{PV}_{0} \quad \text { at } t \geq t_{0}
\end{gathered}
$$

where $\mathrm{S}_{0}$ and $\mathrm{PV}_{0}$ are the initial concentration of the lipid substrate and PV, respectively. From the initial estimations, we found that $\mathrm{S}_{0}$ could be fixed at $42 \mathrm{meq} / \mathrm{kg}-$ oil .
The kinetic parameters, $k$ and $t_{0}$, were calculated by best fitting the experimental results of Solver in Microsoft $\AA$ Excel 2013.

It can be seen from Fig. 1 and Table 1 that adding $5 \mathrm{mg}$ of C18AsA had no effect on the retardation of lipid autoxidation, because the PV rapidly increased after a very short induction time, almost similar to the case of adding SEC16. A small amount of C18AsA might be immediately depleted after reaction with free radicals, such as alkyl radicals formed from the autoxidation reaction. Upon the addition of $100 \mathrm{mg} \mathrm{C18AsA}$, however, the induction time of lipid oxidation could be increased to $189 \mathrm{~h}$, while the addition of $25 \mathrm{mg}$ C18AsA resulted in the longest induction period $(>264 \mathrm{~h})$. This phenomenon could be explained by the dual activities, i.e., the antioxidant and pro-oxidant activities, of the ascorbic acid moiety of C18AsA. Ascorbic acid has strong reducing power and free radical scavenging effect; therefore, it acts as a good antioxidant. [12] However, at the same time, ascorbic acid can act as a pro-oxidant. The $\mathrm{Fe}^{2+}$ ions added to cookies accelerated the lipid autoxidation via several mechanisms such as producing reactive oxygen and degrading lipid hydroperoxides to lipid peroxyl radicals. [13] After the reaction, $\mathrm{Fe}^{3+}$ is formed, which can be reduced to $\mathrm{Fe}^{2+}$ by ascorbic acid (Fenton reaction). Therefore, at higher amounts of C18AsA, a greater number of molecules acted as pro-oxidants; therefore, the induction period was shortened. This result disagreed with the behavior of ascorbic acid in simpler systems; for example, Yen et al. [12] found that in deoxyribose assay, ascorbic acid at low concentrations acted as a pro-oxidant by reducing $\mathrm{Fe}^{3+}$ to $\mathrm{Fe}^{2+}$, but at higher concentrations, ascorbic acid acted as a scavenger of hydroxyl radicals. We have previously reported that in linoleic acid oxidation without a metal catalyst, increasing the molar ratio of ascorbic acid or capryloyl ascorbate to linoleic acid from 0 to 0.1 could increase the stability of linoleic

\begin{tabular}{|c|c|c|c|c|c|c|c|}
\hline Additive & $\begin{array}{l}\text { SEC16*2 } \\
(25 \mathrm{mg})\end{array}$ & $\begin{array}{c}\text { AsA }^{* 3}+\text { SEC16 } \\
\text { (25 mg each) }\end{array}$ & $\begin{array}{c}\text { C16AsA }{ }^{* 4} \\
(25 \mathrm{mg})\end{array}$ & $\begin{array}{l}\mathrm{C} 18 \mathrm{AsA}^{* 5} \\
(5 \mathrm{mg})\end{array}$ & $\begin{array}{l}\text { C18AsA } \\
(25 \mathrm{mg})\end{array}$ & $\begin{array}{l}\text { C18AsA } \\
(100 \mathrm{mg})\end{array}$ & $\begin{array}{c}\mathrm{C}^{2} 2 \mathrm{AsA}^{* 6} \\
(25 \mathrm{mg})\end{array}$ \\
\hline Induction period $t_{0}[\mathrm{~h}]$ & 24 & 13 & 44 & 18.6 & $>264$ & 189 & 8.4 \\
\hline Rate constant $k_{1}\left[\mathrm{~h}^{-1}\right]$ & $5.41 \times 10^{-3}$ & $7.48 \times 10^{-3}$ & $4.35 \times 10^{-3}$ & $6.05 \times 10^{-3}$ & & $4.68 \times 10^{-3}$ & $1.02 \times 10^{-1}$ \\
\hline Rate constant $k_{2}\left[\mathrm{~h}^{-1}\right]$ & $3.08 \times 10^{-3}$ & $6.36 \times 10^{-3}$ & $6.26 \times 10^{-3}$ & $4.99 \times 10^{-3}$ & & $3.56 \times 10^{-3}$ & $7.83 \times 10^{-3}$ \\
\hline
\end{tabular}
acid [5]. It should be noted that $k_{1}$ and $k_{2}$ in each system

Table 1 Kinetic parameters estimated for the oxidation of soybean oil added with acyl ascorbate. ${ }^{* 1}$

*1 The amount of soybean oil added to the cookie was $250 \mu \mathrm{L}$ for each sample.

*2 SEC16: palmitoyl sucrose ester $\quad * 3$ AsA: Ascorbic acid

*4 C16AsA: Palmitoyl ascorbate $\quad * 5$ C18AsA: Stearoyl ascorbate

${ }^{*} 6$ C22AsA: Behenoyl ascorbate 
were not much different.

\subsection{Antioxidant ability of acyl ascorbates with different acyl chain lengths}

Acyl ascorbates with different acyl chain lengths (C16AsA, C18AsA, and C22AsA; $25 \mathrm{mg}$ each) were compared for their antioxidant activities (Fig. 2, Table 1.). Both SEC16 and AsA-SEC16 were used for control treatments. The results showed that $\mathrm{C} 18 \mathrm{AsA}$ was the best antioxidant in the tested food model. C16AsA could only extend the induction time $(44 \mathrm{~h})$ marginally, while C22AsA and AsA-SEC16 did not show any effect on retarding the oxidation. Compared with SEC16, which does not contain an ascorbic moiety, C16AsA, C22AsA, and AsA-SEC16 resulted in faster degradation of PV $\left(k_{2}\right)$. This might be caused by pro-oxidant activity of ascorbic acid, as discussed in a previous section. Direct comparison of the antioxidant activities of C16AsA, C18AsA, and $\mathrm{C} 22 \mathrm{AsA}$ has not been reported in the literature. However, we have reported that the acyl chain length of the ascorbates affects the retardation of lipid autoxidation in different systems. For example, increasing the acyl chain length from 12 to 16 decreased the oxidation of bulk linoleic acid [5]. Caproyl ascorbate was better than capryloyl, lauroyl, and palmitoyl ascorbates for the retardation of encapsulated oil [14]. From these literature data, it can be concluded that the matrix of each sys-



Fig. 2 Changes in peroxide values (PV) of soybean oil (250 $\mu \mathrm{L})$ with time for the cookies with $25 \mathrm{mg}(\square)$ palmitoyl ascorbate (C16AsA), $(\bigcirc)$ stearoyl ascorbate (C18AsA), and $(\triangle)$ behenoyl ascorbate (C22AsA). The changes in the PVs of linoleic acid for the cookie added with $25 \mathrm{mg}$ palmitoyl sucrose ester (SEC16) $(\boldsymbol{\nabla})$, and $25 \mathrm{mg} \mathrm{SEC16}$ and $25 \mathrm{mg}$ ascorbic acid (AsA) $(\nabla)$ were observed as controls. The solid curves were calculated using the estimated kinetic parameters. tem has an influence on the antioxidant activity of each acyl ascorbate. In this study, for example, behenoyl ascorbate, which has slightly higher hydrophobicity than palmitoyl and stearyl ascorbates, might interact with saturated lipids from butter instead of unsaturated lipids from soybean oil, which renders it unavailable for retarding autoxidation.

\section{Acknowledgement}

Pramote Khuwijitjaru gratefully acknowledges the Visiting Research Scholarship from Graduate School of Agriculture, Kyoto University.

\section{References}

1) F. Shahidi, Y. Zhong; Lipid oxidation and improving the oxidative stability. Chem. Soc. Rev. 39, 4067-4079 (2010).

2) Y. Watanabe; Antioxidative properties of acyl ascorbates on lipid oxidation in bulk, disperse and microcapsule systems. Food Sci. Technol. Res. 21, 639-647 (2015).

3) M. Aleman, R. Bou, A. Tres, J. Polo, R. Codony, F. Guardiola; Oxidative stability of a heme iron-fortified bakery product: Effectiveness of ascorbyl palmitate and co-spray-drying of heme iron with calcium caseinate. Food Chem. 196, 567576 (2016).

4) L. Zou, C. C. Akoh; Oxidative stability of structured lipidbased infant formula emulsion: Effect of antioxidants. Food Chem. 178, 1-9 (2015).

5) Y. Watanabe, E. Ishido, X. Fang, S. Adachi, R. Matsuno; Oxidation kinetics of linoleic acid in the presence of saturated acyl L-ascorbate. J. Am. Oil Chem. Soc. 82, 389-392 (2005).

6) Y. Watanabe, H. Nakanishi, N. Goto, K. Otsuka, T. Kimura S. Adachi; Antioxidative properties of ascorbic acid and acyl ascorbates in ML/W emulsion. J. Am. Oil Chem. Soc. 87, 1475-1480 (2010).

7) Y. Watanabe, X. Fang, S. Adachi; Suppressive effect of decanoyl ascorbate on the oxidation of fish oil encapsulated with a polysaccharide. Food Sci. Technol. Res. 15, 569-574 (2009).

8) Y. Watanabe, K. Kuwabara, S. Adachi, K. Nakanishi, R. Matsuno; Production of saturated acyl L-ascorbate by immobilized lipase using a continuous stirred tank reactor. J. Agric. Food Chem. 51, 4628-4632 (2003).

9) E. Løvaas; A sensitive spectrophotometric method for lipid hydroperoxide determination. J. Am. Oil Chem. Soc. 69, 777-783 (1992)

10) W. W. Nawar, Lipids. in: O. R. Fennema, (Ed.), Food 
Chemistry, Marcel Dekker, New York, 1996, pp. 225-319.

11) S. Iibuchi; Analysis of the autoxidation rate of edible oils. Nippon Nogeikagaku Kaishi 70, 29-36 (1996).

12) G.-C. Yen, P.-D. Duh, H.-L. Tsai; Antioxidant and pro-oxidant properties of ascorbic acid and gallic acid. Food Chem. 79, 307-313 (2002).

13) E. Choe, D. B. Min; Mechanisms and factors for edible oi oxidation. Compr. Rev. Food Sci. Food Saf. 5, 169-186 (2006).

14) Y. Watanabe, X. Fang, Y. Minemoto, S. Adachi, R. Matsuno; Suppressive effect of saturated acyl L-ascorbate on the oxidation of linoleic acid encapsulated with maltodextrin or gum arabic by spray-drying. J. Agric. Food Chem. 50, 3984-3987 (2002). 